\title{
Emergency peripartum hysterectomies at Muhimbili National Hospital, Tanzania: a review of cases from 2003 to 2007
}

\author{
ANDREA B. PEMBE*, PETER J.T. WANGWE and SIRIEL N. MASSAWE \\ Department of Obstetrics and Gynaecology, School of Medicine, Muhimbili University of Health and \\ Allied Sciences, P.O. Box 65117, Dar es Salaam, Tanzania
}

\begin{abstract}
A retrospective review of all cases of emergency peripartum hysterectomy performed between January 1, 2003 and December 31, 2007 at Muhimbili National Hospital was done to determine the incidence, indications and complications, background characteristics, antenatal care attendance, referral status, and maternal and foetal outcomes. There were 55,152 deliveries during the study period and 165 cases of emergency peripartum hysterectomy, giving the incidence of emergency peripartum hysterectomy of 3 per 1000 deliveries. The main indication was uterine rupture $(79 \%)$ followed by severe post-partum haemorrhage due to uterine atony $(12.7 \%)$. The case fatality rate was $10.3 \%$ where as perinatal mortality rate was 7.7 per 1000 deliveries. The common complication identified intraoperatively was severe haemorrhage which accounted for $39.4 \%$ where as intensive care unit admissions $(14.4 \%)$ and febrile morbidity $(12.4 \%)$ were common after the operation. Blood was ordered in all cases but in 31 cases it was indicated that it was not available. Seventy nine patients received blood transfusion with the maximum number of units given to one patient being eight. Twenty two patients were given fresh frozen plasma (FFP), the median number of units given was two (range $=1-6$ ). In conclusion, emergency peripartum hysterectomy is a life saving procedure and very common at $\mathrm{MNH}$. The most common indication was ruptured uterus followed by severe postpartum haemorrhage. More than half of the patients underwent emergency peripartum hysterectomy were referred from other health facilities with ruptured or suspected ruptured uterus. The procedure was associated with unacceptably high maternal and perinatal morbidity and mortality.
\end{abstract}

Key words: Emergency, peripartum hysterectomy, uterine rupture, maternal, morbidity, mortality, Tanzania

\section{Introduction}

Emergency peripartum hysterectomy involves removal of the uterus after vaginal delivery or during the caesarean section. The operation is commonly made without delay when a decision is reached to save the life of the mother when conservative methods have failed and the mother's condition is deteriorating. The incidence of emergency peripartum hysterectomy varies from 2 to 10 per 1000 deliveries (Engelsen et al., 2001; Okogbenin et al., 2003; Zamzami, 2003; El-Jallad et al., 2004; Kwame-Aryee et al., 2007). Some studies have reported excessive haemorrhage in the third stage of labour due to uterine atony or placenta praevia as the common indication for emergency peripartum hysterectomy (Zamzami, 2003; Ezechi et al., 2004) while other studies have

*Correspondence: Andrea B. Pembe; E-mail: andreapembe@yahoo.co.uk 
shown ruptured uterus to be the common indication (Chew \& Biswas, 1998; Okogbenin, et al., 2003).

Tanzania is among the countries with a high maternal mortality ratio, currently estimated at 454 per 100,000 live births (TDHS, 2010). A study in Dar es Salaam city by Urassa et al. (1995) found that the major causes of the maternal deaths were haemorrhage, postpartum infection, hypertensive disorders, obstructed labour and complications of abortion. The majority of the deaths occurred in women who had contact to the modern health care system and spent reasonable time receiving treatment. This could suggest inadequate quality of services provided by the health care system. The incidence, indications and complications of emergency peripartum hysterectomy may reflect the quality of antenatal, intrapartum and referral care provided in the country. We conducted a five year review of cases of emergency peripartum hysterectomy at the tertiary and university teaching hospital in Dar ea Salaam, Tanzania, in order to describe the incidence, indications, complications, background characteristics, antenatal care attendance, referral status, and maternal and foetal outcomes.

\section{Material and Methods}

\section{Study setting and design}

This was a retrospective review of all cases of emergency peripartum hysterectomy performed between January 1, 2003 and December 31, 2007 at Muhimbili National Hospital (MNH). MNH is the largest referral and University teaching Hospital in Tanzania. The hospital is located in Dar-es-Salaam city which has an estimated population of 2.5 million (2002 census). The city has three municipal (district) hospitals, 16 private hospitals, 10 health centres and more than 60 dispensaries which are government and non-government owned.

$\mathrm{MNH}$ provides antenatal care in the clinics conducted from Monday to Friday. In average 40 pregnant mothers attend the clinic in a day. The hospital has a maternity building which has seven wards. Four wards each with 40 beds capacity are for antenatal and postnatal mothers. The other three wards are the labour ward, eclampsia ward and the last ward save for neonatal care. The hospital has one intensive care unit (ICU) with 12 beds. Patients needing intensive care are transferred to the unit after interdepartmental consultation of the doctors in the obstetrics and gynaecology and those in the ICU under the department of Anaesthesia.

$\mathrm{MNH}$ attends all pregnant women, received and not received antenatal care at the hospital irrespective of their clinical state. It receives women referred from the municipal hospitals, district hospitals surrounding Dar-es-Salaam city, private hospitals, health centres and dispensaries. Some pregnant mothers are coming directly from home. The annual total number of deliveries is about 11,000. Most obstetrical surgical procedures including hysterectomies are performed by the residents in obstetrics and gynaecology under supervision of the consultants. During the study period MNH was undergoing major rehabilitation and there was one obstetric theatre which was used for both emergency and elective cases. In circumstances when there were too many obstetric 
cases the gynaecological theatre had to be used thus gynaecological procedures were halted.

\section{Data collection}

In this review emergency peripartum hysterectomy was defined as hysterectomy performed after delivery of the baby because of obstetric complication. The register, report books and patients' clinical records in the obstetric theatre and postnatal wards were searched to identify cases of emergency peripartum hysterectomy. The first author and one research assistant extracted information from the patient's clinical records. The information retrieved includes maternal age, parity, gestational age at delivery, presence of uterine scar, mode of delivery, type of hysterectomy whether sub-total or total, as well as complications of the procedure such as intra-operative hypotension, mean blood loss, blood transfusion and intraoperative and postoperative complications. The indication of emergency peripartum hysterectomy was obtained in the operative notes.

\section{Data analysis}

Data entry was done using Epi-Info 6 computer programme and subsequently analysed by Statistical Package for Social Sciences (SPSS) version 14.0. Frequency distribution and measure of location were used to summarize data.

\section{Ethical considerations}

Ethical clearance was given by the Senate, Research and Publication Committee of Muhimbili University of Health and Allied Sciences (MUHAS) and permission to conduct the study was given by the Executive Director of MNH. Retrieval of information was conducted confidentially by researchers and no name of the patients was used in the data collection protocol.

\section{Results}

During the five year period there were 55,152 deliveries and 165 cases of emergency peripartum hysterectomy of which 151 (92\%) were total - and $14(8 \%)$ were sub-total abdominal hysterectomy. Among the 165 cases, 28 were performed in women who had vaginal deliveries, 43 after caesarean section and 94 during laparatomy due to ruptured uterus. The overall incidence of emergency peripartum hysterectomy was 3 per 1000 deliveries. The annual incidence of emergency peripartum hysterectomy increased from 2 per 1000 deliveries in the year 2003 to 3.5 per 1000 in 2004, thereafter there was no much change in the incidence between 2004 to 2007 (Figure 1). 


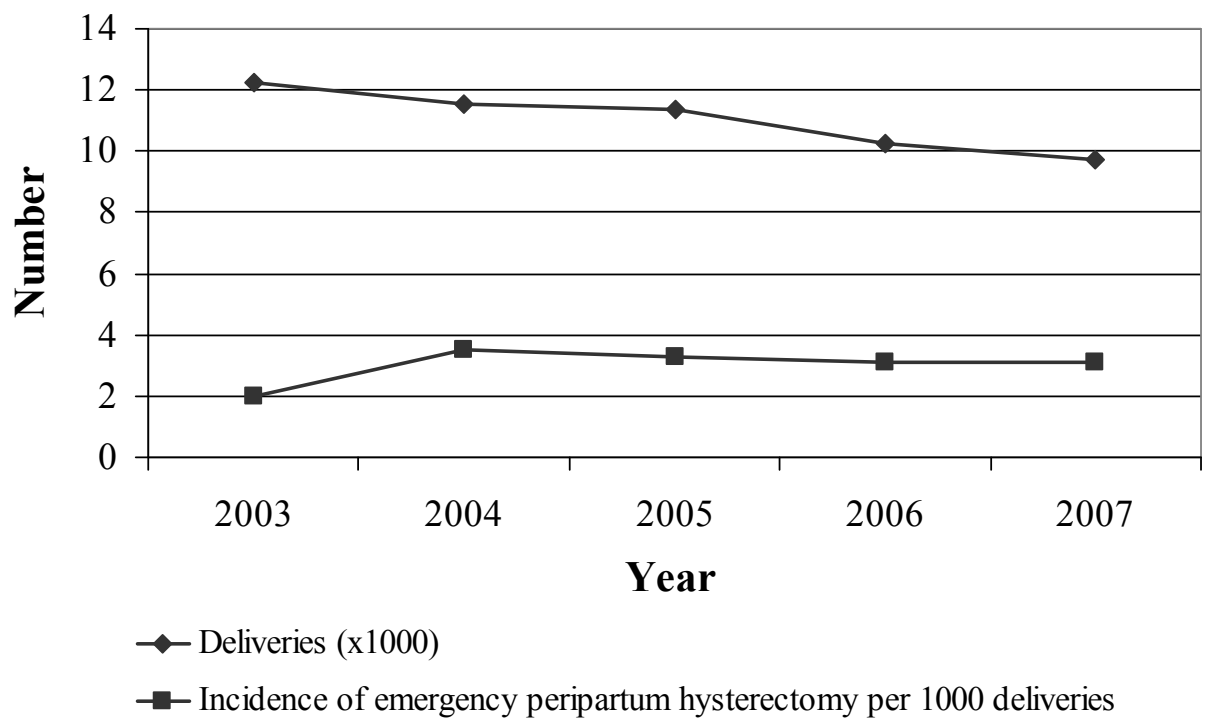

Figure 1: Total deliveries and annual incidence of emergency peripartum hysterectomy

The median age of the cases was 30 years (range $=15-40$ ). The median parity was 3 (range $=1-12$ ). The mean gestation age at delivery was $38 \pm 1.96$ weeks. Eleven per cent of the cases had previous caesarean section scar (Table 1).

Table 1: Demographic, obstetrics and referral characteristics of emergency peripartum hysterectomy cases $(\mathrm{N}=165)$

\begin{tabular}{lll}
\hline Characteristic & Frequency & Percentage \\
\hline $\begin{array}{l}\text { Age group (years) } \\
\quad<20\end{array}$ & 3 & \\
$20-34$ & 126 & 1.8 \\
$\quad 335$ & 36 & 76.4 \\
$\begin{array}{l}\text { Parity } \\
\quad \text { Primiparous }\end{array}$ & 5 & 21.8 \\
$\quad$ Multiparous (2-4) & 120 & 3 \\
$\quad$ Grand multiparous ( $\geq 5)$ & 40 & 72.7 \\
Gestation age (weeks)* & & 24.2 \\
$\quad<36$ & 19 & 11.6 \\
$\quad \geq 37$ & 144 & 88.3 \\
Previous caesarean section & & \\
$\quad$ Yes & 18 & 10.9 \\
$\quad$ No & 147 & 89.1 \\
\hline
\end{tabular}

*Two patients had no gestation age recorded.

Eighty nine (54\%) of the cases were referred to $\mathrm{MNH}$ from other health facilities. The Municipal hospitals contributed majority $(86.6 \%)$ of the referred cases while $7 \%$ cases were referred from the dispensaries and health centres in the Dar es Salaam city. Six per 
cent of the cases referred were from the district hospitals in the neighbouring Coast Region. Among the referred cases, majority were referred due to obstructed labour and suspected ruptured uterus (Table 2).

Table 2: Reasons for referral to $\mathrm{MNH}(\mathrm{N}=89)$

\begin{tabular}{lll}
\hline Reason for referral & Number & Percentage \\
\hline Suspected uterine rupture & 30 & 33 \\
Obstructed labour & 25 & 28 \\
Severe postpartum haemorrhage & 4 & 4.5 \\
Malpresentation & 4 & 4.5 \\
Previous scar & 3 & 3.4 \\
Abruptio placenta & 3 & 3.4 \\
Pregnancy induced hypertension & 2 & 2.2 \\
Anaemia & 2 & 2.2 \\
Others* & 6 & 6.7 \\
Missing & 10 & 11 \\
\hline
\end{tabular}

${ }^{*}$ Others include foetal distress, hydrocephalus, ischemic uterus, placenta praevia, retained placenta and retained second twin

The most common recorded indication for emergency peripartum hysterectomy in the referred and not referred cases was ruptured uterus (79\%) followed by uterine atony $(12.7 \%)$, while myoma and placenta preavia were the least each appearing in $0.6 \%$ of all the cases (Figure 2). Among cases not referred $76(46 \%)$, rupture of the uterus and uterine atony were indications for the operation in $53(69.7 \%)$ and $13(13 \%)$ of the cases, respectively".

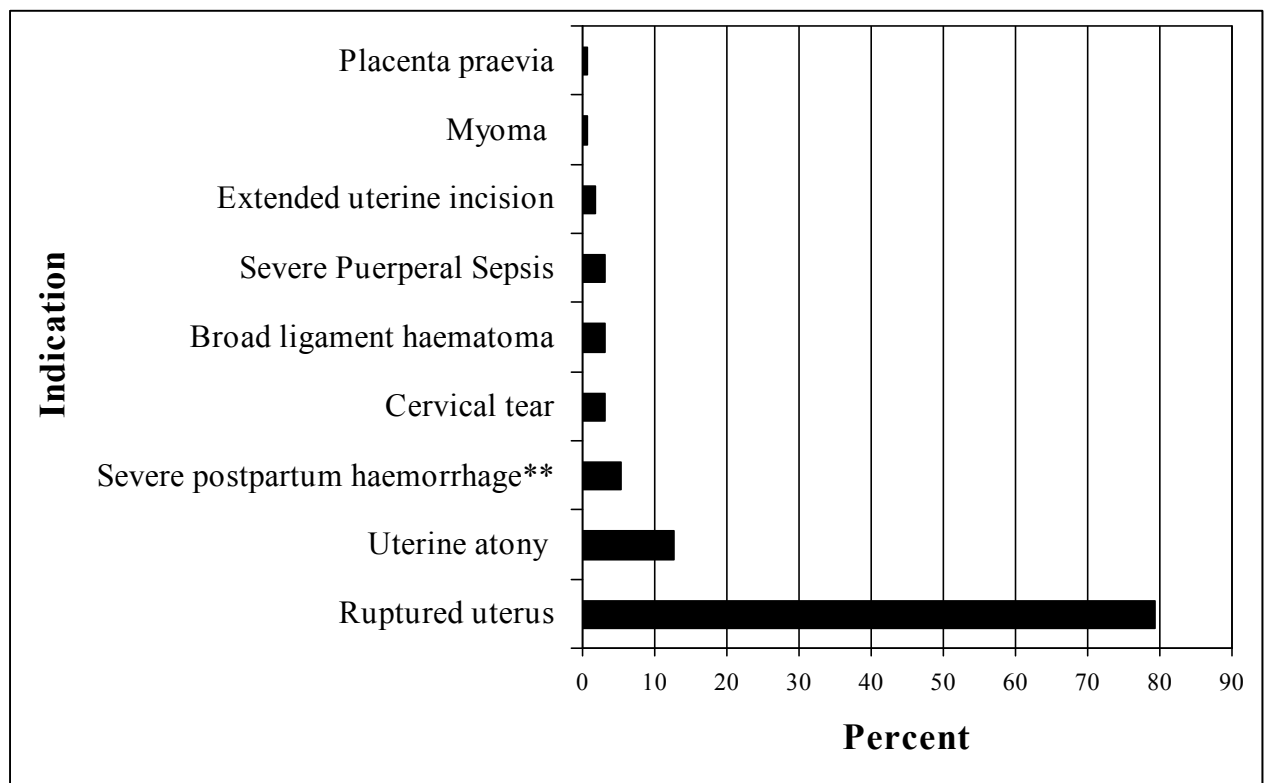

Figure 2: Indications of emergency peripartum hysterectomy $(\mathrm{N}=165)$

**Uterus was well contracted and no clear cause was identified 
Out of 165 patients, 74 (44.8\%) developed intra-operative complications (Table 3). The commonest complication was severe bleeding followed by hypotension. Post-operative complications were experienced by $41(24.8 \%)$ out of 160 patients. Twenty three patients $(14.4 \%)$ were admitted into the intensive care unit (ICU). The commonest complication after the operation was febrile morbidity, defined as a body temperature of $>38{ }^{\circ} \mathrm{C}$ measured at least 24 hours after hysterectomy and repeated at least once.

Table 3: Intra- and post-operative complications among cases of emergency peripartum hysterectomy*

\begin{tabular}{lll}
\hline Complication & Number & Percentage \\
\hline Intra- operative $(\boldsymbol{n}=\mathbf{1 6 5})$ & 65 & 39.4 \\
Severe bleeding & 10 & 6.1 \\
Hypotension & 5 & 3 \\
Cardiac arrest & 2 & 1.2 \\
Bladder injury & 2 & 1.2 \\
Bowel injury & 1 & 0.6 \\
Difficult in breathing & & \\
Post-operative ( $\boldsymbol{n}=\mathbf{1 6 0})^{* * *}$ & 23 & 14.4 \\
ICU admission & 20 & 12.5 \\
Febrile morbidity & 6 & 3.8 \\
Wound sepsis & 5 & 3.1 \\
Septicaemia & 3 & 1.9 \\
Acute renal failure & 3 & 1.9 \\
Peritonitis & 2 & 1.3 \\
Urinary tract infection & 1 & 0.6 \\
Burst abdomen & 1 & 0.6 \\
Coagulopathy & 1 & 0.6 \\
Pneumonia & 1 & 0.6 \\
Re-exploratory laparatomy & 1 & 0.6 \\
Prolonged vaginal bleeding &
\end{tabular}

*Some patients developed more than one complication; ${ }^{* *}$ Five patients died during the operation.

The median blood loss was $1500 \mathrm{ml}$ (range $=300-4000$ ). Blood was ordered in all cases but in 31 cases there was no blood available in the blood bank. Seventy nine cases received blood transfusion of which 35 received one unit each, 23 received two units, 13 received three units and eight received four or more units. The maximum number of blood units given to one patient was eight. Twenty two patients were given fresh frozen plasma (FFP). The median number of units given was two (range $=1-6$ ). There were 18 patients who received both blood and FFP transfusion. Overall, 17 patients died, giving the case fatality rate of $10.3 \%$. Among patients who died, five died during the operation due to cardiac arrest and nine died a few hours after admission to the ICU. Three other patients died in the postnatal wards due to various complications. 


\section{Discussion}

The majority of the cases were referred to the hospital from municipal hospitals within the city and the district hospitals of the neighbouring Coast Region. The common indications for emergency peripartum hysterectomy were ruptured uterus followed by severe post-partum haemorrhage due to uterine atony. The incidence of emergency peripartum hysterectomy in this study did change significantly over the five year period giving an overall incidence of 3 per 1000 deliveries. This incidence is lower than the one reported from a study by Kwame-Aryee et al. (2007) at Korle-Bu teaching hospital in Ghana, but higher than that reported in most studies elsewhere (Zamzami 2003; Okogbenin et al., 2003; El-Jallad et al., 2004; Selo-Ojeme et al., 2005; Katchy et al., 2006; Kwee et al., 2006; Yucel et al., 2006).

The higher incidence of peripartum hysterectomy at Muhimbili may be due to the characteristic of the hospital being a referral institution, thus receiving cases which may need the procedure. The other reason may be inadequate provision of comprehensive emergency obstetric care in the neighbouring municipal hospitals as it is reflected on the total number of patients referred due to obstructed labour and suspected ruptured uterus. These cases should have been operated in the respective hospitals thus decreasing the delays which are associated with the bad outcome including emergency peripartum hysterectomy. It has been observed that there are always long queues of patients awaiting emergency operations in the obstetric theatre. Previous studies have reported some municipal hospitals do not provide 24 hours operative and blood transfusion services, thus it is not surprising that the caesarean rates in the hospitals is 3-4\% while the rate at Muhimbili is more than 32\% (Nyamtema et al., $2008 a, b)$. To improve maternal health there is need to increase and intensify coverage of comprehensive emergency obstetric care services in the city.

Studies in Pakistan and Nigeria report ruptured uterus to be the commonest indication of emergency peripartum hysterectomy (Tahir et al., 2003; Okogbenin et al., 2003). Uterine rupture was an indication in more than three quarters of the emergency peripartum hysterectomy in this study. The much higher percent of ruptured uterus as indicated in this study compared to other developing countries is difficult to explain. It can be speculated that there are more delays either in the patients and their families arriving in the health facilities or the referral process within the health care system or poor monitoring and management in the hospitals. The Dar es Salaam perinatal care study reported that the use of the partogram was poor at Muhmbili and at the neighbouring municipal hospitals (Nyamtema et al., 2008a). Therefore, poor monitoring of women in labour could have contributed to the high number of cases of ruptured uterus.

In most developed countries where availability and accessibility to emergency obstetric care is no longer a problem there is a shift of common indications for emergency peripartum hysterectomy from ruptured uterus to abnormal placentation and uterine atony (Kwee et al., 2006; Knight, 2007). Placenta accrete is the common indication for peripartum hysterectomy in these countries due to increase rate of 
caesarean deliveries. Caesarean section is associated with placentation in the lower segment of the uterus and this increase the risk of morbidly adhered placenta. The rise in the caesarean section rate at Muhimbili (Muganyizi et al., 2008) is alarming as it may increase the cases needing emergency peripartum hysterectomy.

The overall case fatality rate $(10.3 \%)$ in our study is lower than that reported from a study in Nigeria (Okogbenin et al., 2003) but much higher than that reported from Pakistan (Tahir et al., 2003), Turkey (Yucel et al., 2006) and the Netherlands (Kwee et al., 2006). The difference is mostly attributed to quality of care including delayed diagnosis and referral, availability of blood for transfusion, indications and timeliness for hysterectomy. Kwee et al. (2006) and Knight (2007) report that most patients who underwent peripartum hysterectomy received more than 10 units of blood. This amount of blood is rarely available in most hospitals in developing countries. Moreover, ruptured uterus may occur in health care facilities which are not able to provide surgery and blood transfusion thus necessitating referral to comprehensive emergency obstetric care units which may be difficult to access especially in low- and middle income countries.

More than $90 \%$ of emergency peripartum hysterectomies performed in our study were sub-total abdominal hysterectomy (STAH). Relatively lower percentages of STAH have been reported in other studies elsewhere (Kastner et al. 2002; Ezechi, et al., 2004; Selo-Ojeme et al., 2005; Ozden et al., 2005; Rahman et al., 2008). The decision to do total or subtotal emergency hysterectomy depends on the experience of the surgeon performing the operation and the condition of the patient. A lower amount of blood loss and short time of operation used in emergency subtotal hysterectomy is recommended for patients in seriously devastated condition (Knight, 2007). Total hysterectomy is recommended in cases of placenta attached to the lower segment as the whole uterus needs to be removed to stop the bleeding (Murta et al., 1993). Another reason put forward is the potential risks for cervical stump to develop malignancy thus needing regular cytology screening and other problems which may occur such as bleeding or abnormal vaginal discharge (Yucel et al., 2006).

The study been retrospective had some limitations. Certain information about the cases could not be found in the records due to poor documentation. These included education level, marital status and occupation of the patients. Furthermore, haemoglobin levels before the operation and at the discharge, and other information possibly to identify the delays were lacking. This limited our analysis to the available information. Another limitation is the lack of histopathological results. In our centre not all hysterectomies performed are sent for histopathological examination. This may be the reason for lack of any case of morbidly adhered placenta as one of the indications. Despite these limitations this study provides useful information for care of the pregnant women within our health system.

In conclusion, in this study we found a high incidence of emergency peripartum hysterectomy at Muhimbili National Hospital. The most common indication was ruptured uterus followed by severe postpartum haemorrhage. More than half of cases of emergency peripartum hysterectomy were referred from other health facilities with 
suspected ruptured uterus. The case fatality rate was high. This calls for strengthening of the provision of intrapartum care and timely obstetric intervention in the hospitals and improving the referral system when deemed necessary for patients to be referred from the municipal to the national hospital. Availability of blood should be improved to save more lives of the mothers. Further systematic audit of cases of peripartum hysterectomy is needed to identify contributing factors.

\section{Acknowledgements}

The authors would like to thank Muhimbili University of Health and Allied Sciences through the Sida/SAREC staff capacity strengthening project for funding this study. We are grateful to the Executive Director of Muhimbili National Hospital for providing permission to conduct the study. We thank Dr. Wilbroad Massawe for his assistance in the retrieval of the data from the case files.

\section{References}

Chew, S. \& Biswas, A. (1998) Caesarean and postpartum hysterectomy. Singapore Medical Journal 39, 9-13.

El-Jallad, M.F., Zayed, F. \& Al-Rimawi, H.S. (2004) Emergency peripartum hysterectomy in Northern Jordan: indications and obstetric outcome (an 8-year review). Achieves of Gynecology and Obstetrics 270, 271-273.

Engelsen, I.B., Albrechtsen, S. \& Iversen, O.E. (2001) Peripartum hysterectomy-incidence and maternal morbidity. Acta Obstetricia et Gynecologica Scandinavica 80, 409-412.

Ezechi, O.C., Kalu, B.K., Njokanma, F.O., Nwokoro, C.A. \& Okeke, G.C. (2004) Emergency peripartum hysterectomy in a Nigerian hospital: a 20-year review. Journal of Obstetrics \& Gynaecology 24, 372-373.

Kastner, E.S., Figueroa, R., Garry, D. \& Maulik, D. (2002) Emergency peripartum hysterectomy: experience at a community teaching hospital. Obstetrics and Gynecology 99, 971-975.

Katchy, K.C., Ziad, F., Al Nashmi, N. \& Diejomaoh, M.F. (2006) Emergency obstetric hysterectomy in Kuwait: a clinic-pathological analysis. Achieves of Gynecology and Obstetrics 273, 360-365.

Knight, M. (2007) Peripartum hysterectomy in the UK: management and outcomes of the associated haemorrhage. BJOG: An international Journal of Obstetrics and Gynaecology 114, 1380-1387.

Kwame-Aryee, R., Kwakye, A. \& Seffah, J. (2007) Peripartum hysterectomies at the Korle-Bu teaching hospital: a review of 182 consecutive cases. Ghana Medical Journal 41, 133-138.

Kwee, A., Bots, M.L., Visser, G.H. \& Bruinse, H.W. (2006) Emergency peripartum hysterectomy: A prospective study in The Netherlands. European Journal of Obstetrics \& Gynecology and Reproductive Biology 124, 187-192. 
Muganyizi, P., Kidanto, H., Kazaura, M. \& Massawe, S. (2008) Caesarean section: trends and associated factors in Tanzania. African Journal of Midwifery and Women's Health 2, 65-68.

Murta, E.F., Carneiro, J.G. \& De Freitas, M.M. (1993) Total hysterectomy versus subtotal hysterectomy: which procedure should be performed during the pregnantpuerperal period? Revista paulista de Medicina 111, 354-358.

TDHS (2010) Tanzania Demographic and Health Survey 2010: Preliminary Report, Dar es Salaam, Tanzania, National Bureau of Statistics (NBS) and ORC Macro.

Nyamtema, A.S., Urassa, D.P., Massawe, S., Massawe, A., Lindmark, G. \& van Roosmalen, J. (2008a) Partogram use in the Dar es Salaam perinatal care study. International Journal of Gynaecology and Obstetrics 100, 37-40.

Nyamtema, A.S., Urassa, D.P., Massawe, S., Massawe, A., Mtasiwa, D., Lindmark, G. \& van Roosmalen, J. (2008b) Dar es Salaam perinatal care study: needs assessment for quality of care. East African Journal of Public Health 5, 17-21.

Okogbenin, S.A., Gharoro, E.P., Otoide, V.O. \& Okonta, P.I. (2003) Obstetric hysterectomy: fifteen years' experience in a Nigerian tertiary centre. Journal of Obstetrics and Gynaecology 23, 356-359.

Ozden, S., Yildirim, G., Basaran, T., Gurbuz, B. \& Dayicioglu, V. (2005) Analysis of 59 cases of emergent peripartum hysterectomies during a 13-year period. Achieves of Gynecology and Obstetrics 271, 363-367.

Rahman, J., Al-Ali, M., Qutub, H.O., Al-Suleiman, S.S., Al-Jama, F.E. \& Rahman, M.S. (2008) Emergency obstetric hysterectomy in a university hospital: A 25-year review. Journal of Obstetrics and Gynaecology 28, 69-72.

Selo-Ojeme, D.O., Bhattacharjee, P., Izuwa-Njoku, N.F. \& Kadir, R.A. (2005) Emergency peripartum hysterectomy in a tertiary London hospital. Achieves of Gynecology and Obstetrics 271, 154-159.

Tahil, S., Aleen, M. \& Akram, S. (2003) Indications and maternal outcome of emergency peripartum hysterectomy. Pakistan Journal of Medical Sciences 19, 182-186.

Urassa, E., Lindmark, G. \& Nystrom, L. (1995) Maternal mortality in Dar es Salaam, Tanzania: Socio-economic, obstetric history and accessibility of health care factors. African Journal of Health Sciences 2, 242-249.

Yucel, O., Ozdemir, I., Yucel, N. \& Somunkiran, A. (2006) Emergency peripartum hysterectomy: a 9-year review. Achieves of Gynecology and Obstetrics 274, 84-7.

Zamzami, T.Y. (2003) Maternal and perinatal outcome of massive postpartum hemorrhage: a review of 33 cases. Annals of Saudi Medicine 23, 135-139. 